\title{
Análise térmica e propriedades mecânicas de resíduos de polietileno de alta densidade (PEAD)
}

\section{Thermal analysis and mechanical properties of high-density polyethylene (HDPE) scraps}

\author{
Helson Moreira da Costa ${ }^{1,2 *}$, Valéria Dutra Ramos ${ }^{2}$, Mônica Calixto de Andrade ${ }^{1}$ e \\ Paola da Silva Richter Quintana Nunes ${ }^{1}$ \\ 'Instituto Politécnico, Universidade do Estado do Rio de Janeiro - UERJ, Nova Friburgo, RJ, Brasil \\ ${ }^{2}$ Universidade Estácio de Sá - UNESA, Nova Friburgo, RJ, Brasil \\ *hmcosta@iprj.uerj.br
}

\begin{abstract}
Resumo
Embalagens de polietileno de alta densidade (PEAD) pós-consumo foram coletadas e moídas. Após processamento em extrusora de rosca dupla, o material definido como PEAD reciclado (mistura de embalagens brancas, marfins e incolores) foi injetado e as propriedades mecânicas de resistência à tração e resistência ao impacto foram avaliadas e comparadas com uma amostra de PEAD comercial. A análise térmica, através da calorimetria exploratória diferencial (DSC), a microscopia eletrônica de varredura (MEV) e a análise estatística dos resultados experimentais também foram conduzidas. Em relação à resistência à tração, a diferença entre as amostras de PEAD reciclado e PEAD comercial foram tão pouco expressivas que, dentro das condições experimentais adotadas, se pôde afirmar que o material reciclado é equivalente ao comercial. Por sua vez, o modelo cinético aplicado na análise térmica revelou que a amostra de PEAD comercial demonstra um processo de nucleação e crescimento dos cristais mais homogêneo e simples, embora a energia de ativação seja consideravelmente maior do que o das demais amostras de PEAD.
\end{abstract}

Palavras-chave: polietileno de alta densidade, propriedades mecânicas, análise térmica.

\begin{abstract}
High density polyethylene (HDPE) post-consumption bottles were collected and milled. After processing in a twin screw extruder, material defined as recycled HDPE (mixture of white, ivory and colorless bottles) was injection molded and mechanical properties, such as tensile strength and impact strength, were evaluated and compared with a commercial HDPE sample. Thermal analysis by differential scanning calorimetry (DSC), scanning electron microscopy (SEM) and statistical analysis of the experimental results were also carried out. Regarding tensile strength, difference between samples of recycled HDPE and commercial HDPE was so insignificant that, within the adopted experimental conditions, one might say that recycled and commercial materials are equivalent. In turn, kinetic model used in the thermal analysis showed that commercial HDPE presents process of nucleation and growth of crystals more simple and homogeneous, while activation energy is significantly higher than the other samples of HDPE.
\end{abstract}

Keywords: high-density polyethylene, mechanical properties, thermal analysis.

\section{Introdução}

Atualmente, uma grande parte dos resíduos descartados rotineiramente no Brasil é composta por material plástico. Entre os termoplásticos de maior volume está o polietileno de alta densidade (PEAD), muito comum no lixo doméstico. $\mathrm{O}$ aumento do uso, juntamente com o aumento da produção desse material, gerou uma quantidade significativamente grande, a qual está presente em lixões e/ou aterros sanitários ${ }^{[1,2]}$. A crescente conscientização das empresas quanto ao problema vem trazendo benefícios ao meio ambiente. Uma das estratégias adotadas é a reintrodução do descarte ou resíduo polimérico primário, transformando-o

em um produto de menor valor ou destinado a uma mesma aplicação através da reciclagem. No entanto, durante a reciclagem podem ocorrer alguns problemas que diminuem as propriedades finais alcançadas pelo artefato (diminuição do alongamento na ruptura, diminuição da resistência ao impacto, descoloração, etc.) e/ou que dificultam o reprocessamento $^{[1,2]}$. Em investigação anterior ${ }^{[3]}$, diversos resíduos incolores ou coloridos pós-consumo de polietileno de alta densidade (PEAD) foram caracterizados através do índice de fluidez (MFI) e da calorimetria exploratória diferencial (DSC). Modelos cinéticos adequados foram utilizados para 
descrever a cinética de cristalização não-isotérmica das amostras. Foi verificado que os pigmentos presentes no PEAD tinham diferentes habilidades nucleantes durante $o$ processo de cristalização, bem como diferentes efeitos sobre a taxa de cristalização e a energia de ativação necessária para o transporte de segmentos macromoleculares para a superfície do cristal em crescimento. Neste estudo, apenas embalagens pós-consumo incolores, brancas e marfins foram moídas e processadas em conjunto. A análise térmica dos resíduos individuais e do material resultante da extrusão conjunta (PEAD reciclado), assim como a determinação das propriedades mecânicas - resistência à tração e resistência ao impacto - foi realizada. A microscopia eletrônica de varredura (MEV) também foi utilizada para a análise da fratura dos corpos de prova. Para fins de comparação com o material reciclado, uma amostra de PEAD comercial também foi investigada.

\section{Materiais e Métodos}

\subsection{Material}

Os resíduos de PEAD foram escolhidos em função do quantitativo percentual em massa verificado na Central de Coleta Seletiva da Empresa Brasileira de Meio Ambiente (EBMA) situada na cidade de Nova Friburgo, região serrana do estado do Rio de Janeiro. Em função do valor de mercado, embalagens pós-consumo não coloridas de PEAD foram selecionadas baseando-se na codificação numérica. Nesse código, os símbolos são constituídos por um elemento comum, formado por três setas inseridas em um triangulo e apontadas em sentido horário, e um código numérico valor 2 para o PEAD. Após a coleta, as embalagens foram lavadas em água corrente com detergente líquido. Tampas, rótulos e resíduos de cola foram retirados. Posteriormente, as embalagens foram secas ao ar e granuladas. Os grânulos foram mantidos por 30 minutos em contato com uma solução $1 \%$ de hidróxido de sódio conforme procedimento descrito por Remédio e colaboradores ${ }^{[4]}$. Após enxague até $\mathrm{pH}$ neutro, os grânulos foram separados por cor e colocados em estufa para secagem. Finalmente, através do procedimento de partição, os grânulos foram dispostos para as análises do índice de fluidez (MFI), o qual foi determinado segundo a norma ASTM D1238 usando-se o equipamento Melt Flow Quick Index, marca Instron (Ceast Division).

Após a análise térmica por DSC, os resíduos de PEAD brancos, marfins e incolores granulados foram misturados, em proporções equivalentes em massa, e extrusados em extrusora rosca dupla corrotacional interpenetrante, marca Teck Tril, modelo DCT20, com $20 \mathrm{~mm}$ de diâmetro e $\mathrm{L} / \mathrm{D}=36$. O material resultante dessa mistura de resíduos foi designado como PEAD reciclado. Após extrusão e granulação, os corpos de prova para a caracterização dos materiais (ensaios de resistência à tração e ao impacto) foram obtidos por injeção em uma injetora Arburg, modelo Allrounder 270 S400-170. O perfil de temperatura empregado foi de 150 a $180^{\circ} \mathrm{C}$, para a perfeita plastificação do material extrusado, com temperatura no molde de $60^{\circ} \mathrm{C}$, mantida por um termorregulador a óleo. A velocidade (vazão) de injeção foi de $30 \mathrm{~cm}^{3} / \mathrm{s}$.

\subsection{Propriedades mecânicas e microscopia}

Após o processamento e a injeção dos corpos de prova de PEAD reciclado e de PEAD resina comercial, o ensaio de resistência à tração foi conduzido de acordo com a norma ASTM D638 usando-se uma célula de carga de $5 \mathrm{kN}$ e velocidade de aplicação da carga de $25 \mathrm{~mm} / \mathrm{min}$. O módulo de elasticidade (E), o limite de escoamento $\left(\sigma_{\mathrm{e}}\right)$, o limite de resistência à tração $\left(\sigma_{\mathrm{u}}\right)$, o limite de ruptura $\left(\sigma_{\mathrm{f}}\right)$, a deformação na ruptura $\left(\varepsilon_{\mathrm{f}}\right)$ e o módulo de tenacidade $\left(\mathbf{U}_{\mathbf{t}}\right)$ foram os parâmetros obtidos através do ensaio em questão. Após condicionamento por $24 \mathrm{~h}$ em $25{ }^{\circ} \mathrm{C}$, dez corpos de prova de cada amostra foram ensaiados e a média e o desvio-padrão dos resultados foram reportados. Uma máquina de ensaios universal, marca Shimadzu, modelo AUTOGRAPH AG-I 100 kN, foi utilizada. A norma internacional ASTM D256 (Método A) foi usada para o teste de resistência ao impacto Izod (entalhe em V). Após o condicionamento dos corpos de prova em $25^{\circ} \mathrm{C}$ por 24 horas, aproximadamente dez corpos foram ensaiados de cada amostra (PEAD reciclado e PEAD virgem) usando-se uma massa de 5,16 kg para o martelo. A média e o desvio-padrão foram, então, calculados e reportados. A máquina utilizada para o ensaio de impacto instrumentado foi da marca Instron, modelo CEAST 9050. Os corpos de provas das amostras de PEAD comercial e PEAD reciclado, após o ensaio mecânico de resistência ao impacto, tiveram a superfície de fratura analisada através de microscopia eletrônica de varredura (MEV). O microscópio usado foi o MEV da marca Hitashi, modelo TM3000. A superfície de fratura foi analisada sem uso de cobertura metálica e com uma voltagem de $15 \mathrm{kV}$.

\subsection{Análise térmica}

Os diferentes resíduos de PEAD foram analisados através da técnica de calorimetria exploratória diferencial (DSC) utilizando-se o equipamento Perkin Elmer, modelo STA-6000, Silmutaneous Thermal Analyzer. Entre $20 \mathrm{mg}$ a $25 \mathrm{mg}$ de cada amostra foram usados. Cápsulas de porcelana foram utilizadas e, sob atmosfera de ar sintético, o procedimento de análise ocorreu mediante quatro ciclos simultâneos: (i) aquecimento da amostra da temperatura ambiente até $210{ }^{\circ} \mathrm{C}$ com uma taxa de $40{ }^{\circ} \mathrm{C} / \mathrm{min}$; (ii) Isoterma em $210^{\circ} \mathrm{C}$ por 3 minutos; (iii) Resfriamento de $210^{\circ} \mathrm{C}$ até temperatura ambiente com taxas de 5, 10, 15 ou $20^{\circ} \mathrm{C} / \mathrm{min}$; (iv) Aquecimento da temperatura ambiente até $210^{\circ} \mathrm{C}$ com taxas de $5,10,15$ ou $20^{\circ} \mathrm{C} / \mathrm{min}$. Antes de cada análise, para fins de calibração do equipamento, um padrão de índio (In) foi utilizado. Parâmetros de interesse como a temperatura de cristalização $\left(T_{c}\right)$; a temperatura de fusão ( $\mathrm{T}_{\mathrm{m}}$ - correspondente ao segundo aquecimento); a entalpia de cristalização $\left(\Delta \mathrm{H}_{\mathrm{c}}-\right.$ medida da área sob a exoterma); a entalpia de fusão $\left(\Delta \mathrm{H}_{\mathrm{m}}-\right.$ medida da área sob a endoterma); o grau de cristalinidade $\left(\chi_{c}\right.$ - calculado em relação ao PEAD 100\% cristalino); e, outros valores específicos para os modelos cinéticos escolhidos, foram obtidos a partir dos termogramas com auxílio do programa Pyris Thermal Analysis $^{\text {T巛® }}$, versão 10.1. 


\section{Resultados e Discussão}

\section{1 Índice de fluidez (MFI)}

Define-se o índice de fluidez (MFI) como sendo a quantidade em gramas de polímero, que flui durante dez minutos através de um orifício calibrado, em condições de força e de temperatura definidas. Na Tabela 1 são apresentados os valores de MFI das diferentes amostras de polietileno de alta densidade (PEAD)

A análise de variância (ANOVA) pode ser usada na investigação de situações nas quais existem diversas variáveis independentes. Quando são violadas de forma importante as pressuposições de normalidade e homocedasticidade, não se pode confiar no resultado de uma análise de variância tradicional, pois a probabilidade de se cometer um erro do Tipo I afasta-se marcadamente de $\alpha$. A alternativa não-paramétrica para a ANOVA a um critério é o teste de Kruskal-Wallis. O teste de Kruskal-Wallis é o teste não-paramétrico utilizado na comparação de três ou mais amostras independentes. Ele nos indica se há diferença entre pelo menos dois deles. A aplicação do teste utiliza os valores numéricos transformados em postos e agrupados em um só conjunto de dados. A comparação dos grupos é realizada por meio da média dos postos (posto médio) ${ }^{[6,7]}$. Desta forma, com auxílio dos programas SigmaPlot ${ }^{\mathrm{TM} \circledast} 12.3$ e Minitab $^{\mathrm{TM} \circledast} 16$, o teste $H$ de Kruskal-Wallis foi conduzido. As diferenças encontradas entre os valores médios de MFI das amostras de PEAD foram grandes o suficiente para excluir a probabilidade de serem atribuídas a uma variabilidade aleatória, ou seja, há diferença estatística significativa em um nível de confiança de $99 \%$ ( $H=10,026$ com 4 graus de liberdade, $\mathrm{p}=0,040)$. Um teste de comparações múltiplas versus um grupo de controle (método de Dunnett) foi, então, conduzido ${ }^{[6,7]}$. Ainda que as características exatas das diferentes resinas de PEAD não sejam conhecidas (massa molecular e número de ciclos de eventuais reciclagens primárias, por exemplo) pôde-se inferir, mediante o resultado do teste não paramétrico, que o PEAD branco, o qual contém como carga rutilo $\left(\mathrm{TiO}_{2}\right)$, apresentou diferença estatisticamente significativa (nível de confiança de $99 \%$ ) em seu comportamento viscoso.

\subsection{Análise térmica}

Na Tabela 2 são apresentados, resumidamente, os valores encontrados, a partir dos termogramas, para diferentes parâmetros das amostras de PEAD. De um modo geral, todas as amostras apresentaram as mesmas tendências

Tabela 1. Valores do índice de fluidez das diferentes amostras de PEAD

\begin{tabular}{|c|c|c|c|c|c|}
\hline Embalagem & Cor/Agente ${ }^{1}$ & Visual $^{2}$ & Uso & Código & $\begin{array}{c}\text { Índice de fluidez } \\
\text { (g/10 min) }\end{array}$ \\
\hline Produto de limpeza & $\begin{array}{l}\text { Pigment White } \\
6 / \mathrm{TiO}_{2} \text {, rutilo }\end{array}$ & Branco & Álcool comum & $B r$ & $1,69 \pm 0,27$ \\
\hline Produto de limpeza & --- & Incolor $^{3}$ & Amaciante & Inc & $1,27 \pm 0,18$ \\
\hline Produto automotivo & $\mathrm{CaCO}_{3}$ & Marfim & Lubrificante & $M r f$ & $1,09 \pm 0,10$ \\
\hline \multicolumn{4}{|c|}{ Polietileno reciclado (mistura das embalagens pós-consumo) } & $\operatorname{Rec}$ & $1,04 \pm 0,03$ \\
\hline \multicolumn{4}{|c|}{$\begin{array}{l}\text { Resina HD7600U - polietileno de alta densidade, desenvolvido para ser utilizado } \\
\text { em misturas com PEBDL e PEBD para a fabricação de filmes, em especial filmes } \\
\text { termoencolhíveis. Possui controle de géis e aditivação otimizada para esta aplicação. }\end{array}$} & Com & $1,15 \pm 0,01$ \\
\hline
\end{tabular}

${ }^{1}$ Color index e tipo de colorante conforme consulta ${ }^{[5]} .{ }^{2} \mathrm{De}$ acordo com percepção visual. ${ }^{3} \mathrm{PEAD}$ sem pigmentação. ${ }^{4} \mathrm{Condições:} 190{ }^{\circ} \mathrm{C} / 5,00 \mathrm{~kg}$.

Tabela 2. Parâmetros obtidos a partir dos termogramas para as diferentes amostras de PEAD em diferentes taxas de aquecimento/resfriamento.

\begin{tabular}{|c|c|c|c|c|c|c|}
\hline \multirow{2}{*}{ Amostra } & \multicolumn{5}{|c|}{ Parâmetros } & \multirow{2}{*}{$\begin{array}{l}\text { Taxa de aquecimento/ } \\
\text { resfriamento }\left({ }^{\circ} \mathrm{C} / \mathrm{min}\right)\end{array}$} \\
\hline & $\mathrm{T}_{\mathrm{c}}\left({ }^{\circ} \mathrm{C}\right)$ & $\mathbf{T}_{\mathrm{m}}\left({ }^{\circ} \mathrm{C}\right)$ & $\Delta H_{c}(J / g)$ & $\Delta \mathbf{H}_{\mathrm{m}}(\mathrm{J} / \mathrm{g})$ & $\% \chi_{\mathrm{c}}{ }^{1}$ & \\
\hline \multirow{3}{*}{ Incolor } & 116,9 & 137,1 & 254,0 & 203,6 & 69,5 & 5 \\
\hline & 110,7 & 140,7 & 217,7 & 191,3 & 65,3 & 10 \\
\hline & 107,6 & 144,0 & 204,7 & 178,0 & 60,8 & 15 \\
\hline \multirow{3}{*}{ Branco } & 114,6 & 139,3 & 214,5 & 183,0 & 62,5 & 5 \\
\hline & 112,0 & 140,0 & 203,1 & 166,4 & 56,8 & 10 \\
\hline & 105,4 & 145,8 & 187,9 & 154,0 & 52,6 & 15 \\
\hline \multirow{3}{*}{ Marfim } & 115,3 & 133,5 & 179,9 & 170,3 & 58,1 & 5 \\
\hline & 109,7 & 137,9 & 172,9 & 154,8 & 52,8 & 10 \\
\hline & 105,2 & 141,6 & 144,5 & 127,9 & 43,7 & 15 \\
\hline \multirow{3}{*}{ Comercial } & 116,5 & 130,4 & 184,0 & 170,1 & 58,1 & 5 \\
\hline & 114,5 & 130,6 & 183,1 & 167,4 & 57,1 & 10 \\
\hline & 113,7 & 131,9 & 181,3 & 157,1 & 53,6 & 15 \\
\hline \multirow{3}{*}{ Reciclado } & 113,4 & 132,1 & 142,3 & 140,9 & 48,1 & 5 \\
\hline & 109,1 & 141,1 & 135,7 & 134,2 & 45,8 & 10 \\
\hline & 105,9 & 148,7 & 136,3 & 105,8 & 36,1 & 15 \\
\hline
\end{tabular}

${ }^{1}$ Calculado de acordo com: , onde $\Delta \mathrm{H}_{\mathrm{m}}{ }^{\circ} \mathrm{p} / \mathrm{PEAD} 100 \%$ cristalino $=293 \mathrm{~J} / \mathrm{g}$. 
com o aumento das taxas de resfriamento / aquecimento: (i) houve uma contínua redução nos valores encontrados para: a temperatura de cristalização ( $\mathbf{T}_{\mathbf{c}}$ ), a qual é estimada pelo pico da curva exotérmica e corresponde ao máximo valor da taxa de cristalização em cada taxa de resfriamento usada; a entalpia de cristalização $\left(\Delta \mathrm{H}_{\mathrm{c}}\right)$; a entalpia de fusão $\left(\Delta \mathrm{H}_{\mathrm{m}}\right)$; e, o grau de cristalinidade $\left(\% \chi_{\mathrm{c}}\right)$. A magnitude de tal redução dependeu da amostra em particular considerada; e, (ii) houve um deslocamento dos picos das curvas endotérmicas, relativos à temperatura de fusão $\left(\mathbf{T}_{\mathrm{m}}\right)$, para valores mais elevados.

A cristalização é controlada pela nucleação, ou seja, em baixas velocidades de resfriamento há tempo suficiente para os núcleos serem ativados em temperaturas mais altas. Consequentemente, a cristalização é nucleada em temperaturas maiores quando as amostras de polímero são resfriadas em velocidades mais baixas. Ao contrário, em velocidades de resfriamento mais altas a ativação dos núcleos ocorre em temperaturas mais baixas ${ }^{[8,9]}$. Isso explica as tendências observadas, ou seja, a redução dos valores de $\mathbf{T}_{\mathbf{c}}, \Delta \mathrm{H}_{\mathrm{c}}, \Delta \mathrm{H}_{\mathrm{m}} \mathrm{e} \% \chi_{\mathrm{c}}$

Pôde-se observar que houve grande diferença na variação dos valores de $\mathbf{T}_{\mathrm{c}}$ da amostra comercial (resina HD7600U) e do restante das amostras de PEAD pós-consumo (incolor, branco e marfim) e reciclada, sobretudo em taxas de resfriamento maiores. A maior diferença verificada, $\Delta \mathrm{T}_{\mathfrak{c}}$, foi de $8,5^{\circ} \mathrm{C}$, alcançada em taxa de $15{ }^{\circ} \mathrm{C} / \mathrm{min}$ de resfriamento entre as amostras PEAD comercial e PEAD marfim. Embora uma interpretação mais acurada deva ser encontrada na análise dos dados da cinética de cristalização, pôde-se inferir de maneira preliminar que as diferentes amostras de PEAD possuem características distintas no desenvolvimento do processo cristalização. Verificou-se também uma grande diferenciação entre as amostras com relação à $\mathbf{T}_{\mathrm{m}}$. Enquanto a amostra comercial de $\mathrm{PEAD}$ pouco é afetada pela variação da taxa de aquecimento, as demais amostras demonstraram uma variação crescente e praticamente linear entre $a \mathbf{T}_{\mathrm{m}}$ e a taxa de aquecimento. Com relação aos valores de $\Delta \mathrm{H}_{\mathrm{c}}$, pôde ser observado que a amostra de PEAD incolor apresentou os maiores valores de $\Delta \mathrm{H}_{\mathrm{c}}$ em comparação com as demais amostras. Em contrapartida, o PEAD reciclado (mistura dos resíduos pós-consumo) possuiu valores de $\Delta \mathrm{H}_{\mathrm{c}}$ substancialmente menores entre as amostras investigadas valor $44 \%$ inferior em relação ao $\Delta H_{c}$ da amostra de PEAD incolor na taxa de $5{ }^{\circ} \mathrm{C} / \mathrm{min}$, respectivamente. Quanto aos demais parâmetros, o PEAD incolor apresentou os maiores valores de $\Delta \mathrm{H}_{\mathrm{m}}$ e $\% \chi_{\mathrm{c}}$. Por outro lado, a amostra PEAD reciclado demonstrou os menores valores para os parâmetros citados, enquanto PEAD comercial exibe comportamento singular com pouca variação com o aumento da taxa de aquecimento. Uma interpretação efetiva dos parâmetros experimentais determinados a partir dos termogramas, bem como a real influência dos aditivos sobre as amostras de PEAD devem ser alcançadas através da análise da cinética de cristalização.

\subsection{Cinética de cristalização não-isotérmica}

O modelo cinético desenvolvido por Liu et al. ${ }^{[10]}$ foi usado para descrever o processo de cristalização não-isotérmico. Neste modelo, as equações de Avrami e Ozawa são combinadas para um dado valor de cristalinidade relativa, conforme a Equação 1:

$$
\ln =\ln \mathrm{F}(\mathrm{T})-\mathrm{b} \cdot \ln \mathrm{t}
$$

onde: $\mathbf{F}(\mathbf{T})$ refere-se à velocidade de resfriamento do processo; b é a razão entre as constantes de Avrami e Ozawa, ou seja, n / m; e, t é o tempo.

O modelo matemático que permite o cálculo da energia de ativação para cada grau de cristalinidade é descrito segundo a Equação 2 abaixo $^{[11]}$ :

$$
\ln \left(\frac{\mathrm{d}_{\mathrm{t}}}{\mathrm{dt}}\right)_{\mathrm{t}}=\mathrm{C}-\Delta \mathrm{E} / \mathrm{RT}
$$

onde: C é um fator arbitrário pré-exponencial; $\Delta \mathrm{E}$ é a energia de ativação para um dado valor de cristalinidade relativa, $\chi_{i}$; T refere-se a uma série de temperaturas relacionadas a uma dada cristalinidade $\chi_{\mathrm{t}}$ em diferentes taxas de resfriamento; $\mathrm{R}$ é a constante universal dos gases $\left(8,314 \mathrm{~J} \mathrm{~mol}^{-1} \mathrm{~K}^{-1}\right)$; e, $\mathrm{d} \chi_{\mathrm{t}} / \mathrm{dt}$ é a taxa de cristalização instantânea em função do tempo para uma dada cristalinidade relativa $\chi_{t}$.

As temperaturas para determinado valor de $\chi_{t}$ podem ser escolhidas selecionando-se graus de cristalinidade relativa - neste estudo, a faixa de $5 \%$ a $95 \%$ foi escolhida para as amostras de PEAD, a fim de que uma ampla análise do processo de cristalização pudesse ser conduzida.

As Figuras 1 e 2 apresentam os parâmetros $\mathbf{F}(\mathbf{T})$ e b do modelo de Liu obtidos por regressão linear. A representação em gráfico de barras foi usada para dar uma ideia da variação nos valores dos parâmetros, de acordo com a maior ou menor adequação dos dados experimentais.

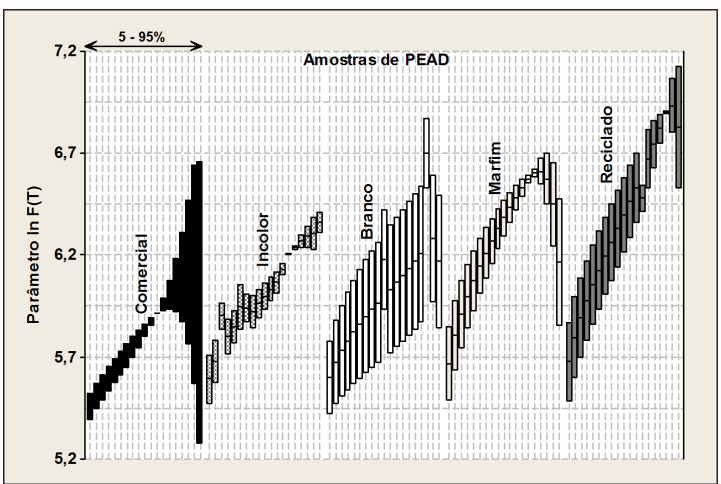

Figura 1. Variação do parâmetro ln $\mathbf{F}(\mathbf{T})$, do modelo cinético de Liu, para as amostras de PEAD.

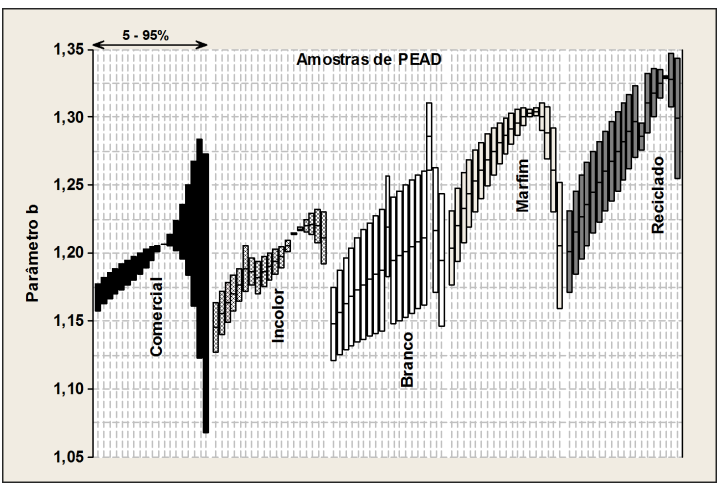

Figura 2. Variação do parâmetro b, do modelo cinético de Liu, para as amostras de PEAD. 
Nas Figuras 1 e 2 percebe-se a dificuldade da adequação do modelo de Liu, sobretudo para as amostras PEAD nos estágios finais de cristalização $\left(\chi_{\leftarrow} \geq 80 \%\right)$ onde há considerável desvio-padrão nos valores experimentais encontrados. Entre as diversas causas possíveis, há de se considerar o mecanismo de cristalização secundária que não é considerado no desenvolvimento do modelo ${ }^{[10]}$. Para um determinado valor de cristalinidade relativa, $\chi_{\mathbf{t}}$, um elevado valor de $\mathbf{F}$ (T) significa que uma taxa de resfriamento alta é necessária para que $\chi_{t}$ seja alcançado na unidade de tempo, ou seja, há uma indicação da dificuldade do processo de cristalização ocorrer.

Através da Figura 1 e da análise estatística (teste $H$ de Kruskal-Wallis - houve diferença estatística significativa em um nível de confiança de $99 \%$ com $H=48,818$ com 4 graus de liberdade e $\mathrm{p} \leq 0,001$ ), verificou-se que: (i) a amostra de PEAD comercial exibiu um comportamento distinto de todas as demais amostras. Embora exista uma inadequação ao modelo de Liu para valores de $\chi_{\mathrm{t}} \geq 80 \%$, há um indício de um processo de cristalização mais homogêneo; (ii) houve uma equivalência entre as amostras de PEAD incolor e PEAD branco e as amostras de PEAD marfim e PEAD reciclado. Do ponto de vista estatístico, em nível de confiança de $99 \%$, o parâmetro $\ln \mathbf{F}(\mathbf{t})$ foi similar entre os pares citados; e, (iii) como o parâmetro $\mathbf{F}(\mathbf{T})$ refere-se à velocidade de resfriamento do processo de cristalização, pôde-se inferir que nos pares citados houve dificuldades similares do processo de cristalização ocorrer.

O parâmetro $\mathbf{b}$ pode ser entendido como um parâmetro ligado à morfologia cristalina das amostras e é definido como a razão entre as constantes de Avrami e Ozawa, ou seja, $\mathbf{n} / \mathbf{m}^{[10]}$. Através da Figura 2 e da análise estatística (houve diferença significativa em um nível de confiança de $99 \%$ com $H=87,506$ com 4 graus de liberdade e $\mathrm{p} \leq 0,001$ ), determinou-se que: (i) o PEAD reciclado foi similar apenas ao PEAD marfim e vice-versa. Do ponto de vista estatístico, os resultados experimentais sugeriram morfologias equivalentes entre as amostras; e, (ii) em contrapartida, as demais amostras de PEAD - comercial, branco e incolor exibiram também similaridades entre si, ou seja, as razões $\mathbf{n} / \mathbf{m}$ das amostras foram equivalentes ao longo do processo de cristalização, $5 \% \leq \chi_{t} \leq 95 \%$. Assim sendo, nos conjuntos citados de amostras de PEAD provavelmente houve etapas de crescimento dos cristais similares, o que proporcionaria uma morfologia final equivalente.

Na Figura 3 são apresentados os valores calculados para a energia de ativação, $\mathbf{E}_{\mathrm{a}}$, necessária para a cristalização não-isotérmica das diferentes amostras de PEAD dentro do intervalo de $5 \%$ a $95 \%$ de cristalinidade relativa $\left(\chi_{t}\right)$. A representação em gráfico de barras mais uma vez foi usada para dar uma ideia da variação no valor do parâmetro, de acordo com a maior ou menor adequação dos dados experimentais. Pôde ser observado que: (i) a amostra de PEAD comercial apresentou os valores mais elevados de $\mathbf{E}_{\mathrm{a}}$ para os estágios iniciais de cristalização $\left(5 \% \leq \chi_{t} \leq 15 \%\right)$, ainda que tais dados também reflitam a dificuldade de adequação dos dados experimentais ao modelo linear proposto; e, (ii) com exceção da amostra de PEAD incolor, em todas as demais

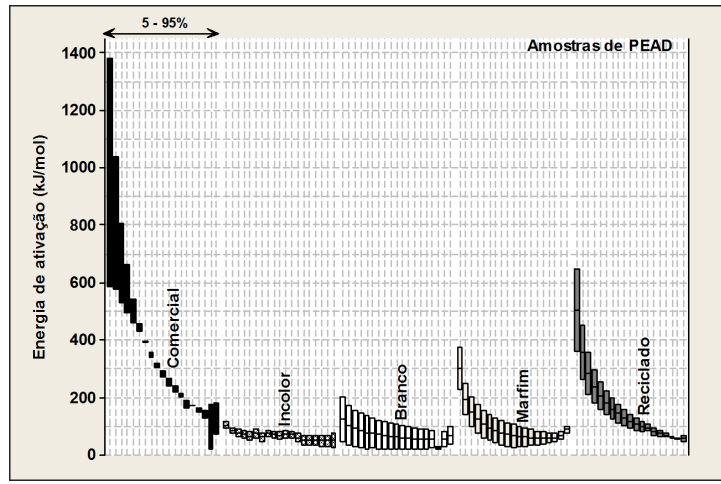

Figura 3. Energia de ativação do processo de cristalização não isotérmico para as diferentes amostras de PEAD na faixa de $5 \leq \chi_{1}(\mathbf{T}) \leq 95 \%$.

houve uma diminuição progressiva da $\mathbf{E}_{\mathrm{a}}$ à medida que $\mathrm{a}$ cristalização atingiu valores mais elevados de $\chi_{\mathbf{t}}$

Rabello $^{[12]}$ afirma que na nucleação heterogênea, a presença de impurezas (como resíduos de catalisadores), cargas ou pigmentos serve como núcleos de cristalização, o que reduz a energia livre crítica para a consolidação do núcleo acelerando o processo global de cristalização. Assim, se na amostra de polietileno comercial, o início do processo de cristalização exigiu um maior gasto energético, talvez seja porque existiu uma maior dificuldade inicial de formação dos primeiros núcleos cristalinos. Por outro lado, a presença das cargas de $\mathrm{TiO}_{2}$ (PEAD branco) e $\mathrm{CaCO}_{3}$ (PEAD marfim) poderia explicar os menores valores de $\mathbf{E}_{\mathrm{a}}$, ainda que não necessariamente tenham sido atingidos valores mais elevados de $\% \chi_{c}$ (ver Tabela 2). Em tais amostras, houve equivalência do ponto de vista estatístico, de acordo com teste post hoc $\mathrm{SNK}^{[6,7]}$, quanto aos valores de $\mathbf{E}_{\mathbf{a}}$, indicando similaridade no processo de cristalização. Por sua vez, no PEAD incolor, resíduo pós-consumo sem pigmentação, características inerentes à resina (massa molecular média, distribuição de massa molecular, entre outras) poderiam explicar os pequenos valores e a pequena variação de $\mathrm{E}_{\mathrm{a}}$ ao longo do processo de cristalização, o que se refletiu em elevado grau de cristalinidade com $65 \% \leq \chi_{\mathrm{c}} \leq 70 \%$ como verificado na Tabela 2. Quanto à amostra denominada PEAD reciclado, a mistura de material granulado de embalagens diversas de polietieno e, consequentemente, a heterogeneidade das resinas, presença de cargas e demais fatores inerentes à reciclagem pós-consumo refletiu-se nos valores de $\mathbf{E}_{\mathrm{a}}$ superiores aos polietilenos incolor, branco e marfim.

\subsection{Propriedades mecânicas}

$\mathrm{Na}$ Tabela 3 encontram-se os valores dos diferentes parâmetros encontrados no ensaio de resistência à tração das amostras de PEAD comercial (HD7600U) e de PEAD reciclado (misturas das embalagens granuladas incolores e de colorações branca e marfim).

Os dados experimentais, em conjunto com a análise estatística, permitiram concluir que os comportamentos mecânicos em tração das amostras de PEAD comercial e PEAD reciclado foram bastante similares, sobretudo quanto ao escoamento $\left(\sigma_{\mathrm{e}}\right)$, ao processo de ruptura $\left(\sigma_{\mathrm{f}}\right.$ e $\left.\varepsilon_{\mathrm{f}}\right)$ e ao 
módulo de tenacidade $\left(\mathbf{U}_{\mathbf{t}}\right)$. Houve apenas um desempenho ligeiramente superior da amostra comercial quanto ao módulo de elasticidade $(\mathbf{E})$ e ao limite de resistência à tração $\left(\sigma_{u}\right)$, os quais foram $4 \%$ e $2 \%$ superiores ao do PEAD reciclado, respectivamente. Em trabalho de Silva ${ }^{[13]}$, o polietileno de alta densidade após ser submetido a quatorze ciclos de extrusão consecutivos ainda apresentou considerável estabilidade em relação às propriedades mecânicas em termos de resistência à tração. A cisão das cadeias poliméricas pela ação conjunta do calor e do cisalhamento não implicou em uma degradação expressiva do material. Na Figura 4 são apresentados os resultados experimentais obtidos para



Figura 4. Gráfico de resistência ao impacto das amostras de PEAD comercial e PEAD reciclado.

(a) PEAD comercial

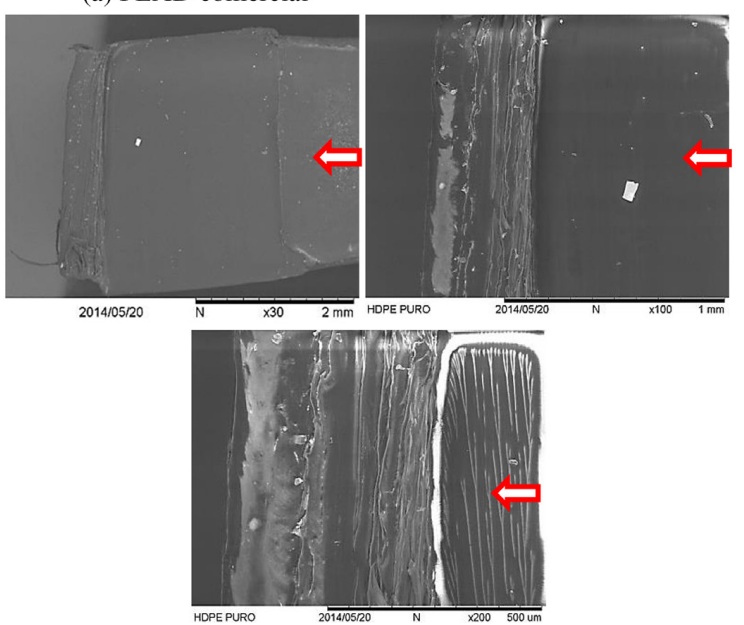

o ensaio de resistência ao impacto Izod das amostras de PEAD comercial e PEAD reciclado. Dentre as diversas variáveis que influenciam o ensaio de impacto pode-se citar as condições de processamento (degradação causada por temperaturas elevadas; formação de linhas de solda, entre outras) e o grau de cristalinidade (o aumento do grau de cristalinidade diminui a resistência ao impacto, podendo ocorrer fratura frágil ${ }^{[14]}$.

Nas fotomicrografias da Figura 5a, o corpo-de-prova da amostra de PEAD comercial apresenta microfibrilas que foram formadas durante a propagação da trinca imposta pelo entalhe. Isso pode ter concedido uma maior resistência ao impacto para a amostra em função de uma fratura mais dúctil. Por outro lado, nas fotomicrografias da Figura 5b, o corpo-de-prova da amostra de PEAD reciclado apresenta uma fratura mais frágil com menor geração de microfibrilas, o que poderia ter ocasionado uma propagação mais rápida da trinca e, consequentemente, uma menor resistência ao impacto. O PEAD reciclado foi resultante do processamento por extrusão e injeção de uma mistura de resíduos - embalagens pós-consumo de colorações branca (carga de $\mathrm{TiO}_{2}$ ), marfim (carga de $\mathrm{CaCO}_{3}$ ) e incolores. A heterogeneidade da composição do reciclado, a mistura de polietilenos contendo teores não conhecidos de cargas minerais diferentes e as eventuais condições de processamento podem ter gerado um material final com desempenho mecânico, aproximadamente, $36 \%$ inferior ao PEAD comercial.

Figura 5. Fotomicrografias da superfície de fratura do corpo de prova de resistência ao impacto. (a) PEAD comercial; (b) PEAD reciclado. A direção de propagação da fratura é indicada pela seta. Aumentos: x 30, x 100 e x 200.

Tabela 3. Propriedades mecânicas em resistência à tração para as amostras de PEAD comercial e PEAD reciclado.

\begin{tabular}{ccccccc}
\hline \multirow{2}{*}{$\begin{array}{c}\text { Amostra de } \\
\text { PEAD }\end{array}$} & $\begin{array}{c}\text { Módulo de } \\
\text { elasticidade E, } \\
\text { MPa }\end{array}$ & $\begin{array}{c}\text { Limite de } \\
\text { escoamento } \boldsymbol{\sigma}_{\mathbf{e}}, \\
\mathbf{M P a}\end{array}$ & $\begin{array}{c}\text { Limite de } \\
\text { resistência à } \\
\text { tração } \\
\boldsymbol{\sigma}_{\mathrm{u}},(\mathbf{M P a})\end{array}$ & $\begin{array}{c}\text { Limite de } \\
\text { ruptura } \boldsymbol{\sigma}_{\mathfrak{f}}, \\
\mathbf{M P a}\end{array}$ & $\begin{array}{c}\text { Peformação na } \\
\text { ruptura } \boldsymbol{\varepsilon}_{\mathrm{f}} \text { (\%) }\end{array}$ & $\begin{array}{c}\text { Módulo de } \\
\text { tenacidade } \mathbf{U}_{\mathrm{t}} \text {, (J) }\end{array}$ \\
\hline $\begin{array}{c}\text { Comercial } \\
\text { HD7600U }\end{array}$ & $826 \pm 18$ & $21,4 \pm 0,2$ & $25,8 \pm 0,2$ & $11,4 \pm 0,3$ & $24,5 \pm 2,2$ & $30,8 \pm 1,6$ \\
Reciclado & $793 \pm 17$ & $21,2 \pm 0,3$ & $25,3 \pm 0,4$ & $7,6 \pm 0,3$ & $21,3 \pm 1,3$ & $27,5 \pm 1,6$ \\
\hline
\end{tabular}




\section{Conclusões}

A análise térmica, através da calorimetria exploratória diferencial (DSC), das amostras, em conjunto com a análise estatística dos resultados experimentais, permitiu concluir que: (i) a amostra de PEAD reciclado, em função de sua heterogeneidade, apresentou os menos valores para os parâmetros referentes à cristalização $\left(\mathbf{T}_{\mathfrak{c}}, \Delta \mathrm{H}_{\mathbf{c}} \mathrm{e} \% \boldsymbol{\chi}_{\mathbf{c}}\right)$; (ii) em contrapartida, o PEAD comercial apresentou um comportamento intermediário entre a amostra de PEAD marfim e as demais usadas nesta investigação.

O modelo cinético aplicado revelou que a amostra de PEAD comercial demonstra um processo de nucleação e crescimento dos cristais mais homogêneo e simples, embora a energia de ativação seja consideravelmente maior do que o das demais amostras.

Em relação às propriedades mecânicas: (i) quanto à resistência à tração, não houve diferença estatística significativa para os parâmetros no escoamento e na ruptura. Mesmo em propriedades como o módulo de elasticidade (E) e o limite de resistência à tração $\left(\sigma_{u}\right)$, a diferença entre as amostras de PEAD reciclado e PEAD comercial foram tão pouco expressivas que, dentro das condições experimentais adotadas, podemos afirmar que o material reciclado é equivalente ao comercial. Quanto ao ensaio de resistência ao impacto, uma diferença expressiva foi encontrada no desempenho da amostra comercial quando comparada à amostra reciclada. Em função das variáveis que afetam o ensaio mecânico, talvez as heterogeneidades do material e as condições de processamento tenham sido responsáveis pelo inferior desempenho do PEAD reciclado.

\section{Agradecimentos}

Os autores agradecem ao CNPq, CAPES e FAPERJ pelo suporte financeiro. Os autores também agradecem ao IMA/UFRJ pelo suporte experimental e ao Programa Bolsista Produtividade da UNESA/2015 fundamental para o desenvolvimento do projeto.

\section{Referências}

1. Mano, E. B., Pacheco, E. B. M. A., \& Bonelli, C. M. C. (2005). Meio ambiente, poluição e reciclagem. São Paulo: Edgard Blücher.

2. Empresa Brasileira de Meio Ambiente-EBMA. Recuperado em 21 de setembro de 2014, de http://www.ebma.com.br
3. Costa, H. M., Andrade, M. C., Lessa, M. D., \& Ramos, V. D. (2014). Cinética de cristalização não-isotérmica de Resíduos de Polietileno de Alta Densidade (PEAD). Polímeros. Ciência e Tecnologia, 24(4), 521-528. http://dx.doi.org/10.1590/01041428.1600.

4. Remédio, M. V. P., Zanin, M., \& Teixeira, B. A. N. (1999). Caracterização do efluente de lavagem de filmes plásticos pós-consumo e determinação das propriedades reológicas do material reciclado. Polímeros. Ciência e Tecnologia, 9(4), 177 183. http://dx.doi.org/10.1590/S0104-14281999000400029.

5. Tríplice Cor. São Paulo. Recuperado em 15 de janeiro de 2014, de http://www.triplicecor.com.br

6. Field, A. (2009). Descobrindo a estatística usando o SPSS (2. ed). Porto Alegre: Bookman.

7. Witte, R. S. \& Witte, J. S. (2005). Estatística (7. ed.). LTC Editora.

8. Tri, P. N., Domenek, S., Guinault, A., \& Sollogoub, C. (2013). Crystallization behavior of poly(lactide)/poly( $\beta$-hydroxybutyrate)/ talc composites. Journal of Applied Polymer Science, 129(6), 3355-3365. http://dx.doi.org/10.1002/app.39056.

9. Blagojević, S. L., Buhin, Z., \& Igrec, I. (2012). Influence of silica nanofiller on the isothermal crystallization and melting of polyurethane elastomer. Journal of Applied Polymer Science, 129(3), 1466-1475. http://dx.doi.org/10.1002/app.38846.

10. Liu, Y., Wang, L., He, Y., Fan, Z., \& Li, S. (2010). Nonisothermal crystallization kinetics of poly(L-lactide). Polymer International, 59(12), 1616-1621. http://dx.doi.org/10.1002/ pi.2894.

11. Saengsuwan, S., Tongkasee, P., Sudyoadsuk, T., Promarak, V., Keawin, T., \& Jungsuttiwong, S. (2011). Non-isothermal crystallization kinetics and thermal stability of the in situ reinforcing composite films based on thermotropic liquid crystalline polymer and polypropylene. Journal of Thermal Analysis and Calorimetry, 103(3), 1017-1102. http://dx.doi. org/10.1007/s10973-010-1022-3.

12. Rabello, M. (2000). Agentes nucleantes. In: M. Rabello. Aditivação de polímeros (cap. 9, pp. 158-165). São Paulo: Artliber.

13. Silva, S. P., Jr. (2010). Análise das propriedades mecânicas do polietileno de alta densidade reciclado (Monografia). Faculdade de Tecnologia da Zona Leste, Centro Paula Souza, São Paulo.

14. Candian, L. M. (2007). Estudo do polietileno de alta densidade reciclado para uso em elementos estruturais (Dissertação de mestrado). Escola de Engenharia de São Carlos, Universidade de São Paulo, São Paulo.

Enviado: Fev. 23, 2015

Revisado: Maio 02, 2015 Aceito: Jul. 22, 2015 